Relations industrielles

Industrial Relations

\title{
Automation: Threat of Promise? - Impact and Implications in Australia, by G.W. Ford, ed., Law Book Co. Ltd, 1969, 214 pp.
}

\section{Laurent Bélanger}

Volume 25, numéro 2, 1970

URI : https://id.erudit.org/iderudit/028138ar

DOI : https://doi.org/10.7202/028138ar

Aller au sommaire du numéro

Éditeur(s)

Département des relations industrielles de l'Université Laval

ISSN

0034-379X (imprimé)

1703-8138 (numérique)

Découvrir la revue

Citer ce compte rendu

Bélanger, L. (1970). Compte rendu de [Automation : Threat of Promise? - Impact and Implications in Australia, by G.W. Ford, ed., Law Book Co. Ltd, 1969, 214 pp.] Relations industrielles / Industrial Relations, 25(2), 382-382.

https://doi.org/10.7202/028138ar

Tous droits réservés (C) Département des relations industrielles de l'Université Laval, 1970
Ce document est protégé par la loi sur le droit d'auteur. L’utilisation des services d'Érudit (y compris la reproduction) est assujettie à sa politique d'utilisation que vous pouvez consulter en ligne.

https://apropos.erudit.org/fr/usagers/politique-dutilisation/ 
With supervisory mobility what it is today, this study illustrates in a specific way, the general problems managers must face when transfers become a way of corporate life.

\section{Joan SICHEL}

\section{Automation: Threat or Promise ? - Im-} pact and Implications in Australia, by G.W. Ford, ed., Law Book Co. Ltd., 1969, 214 pp.

Ce volume est un compte-rendu d'un symposium tenu par l'Association pour l'avancement des sciences de l'Australie et de la Nouvelle-Zélande. L'année exacte n'est pas mentionnée mais il semblerait que le sous-comité sur l'automation ait commencé son travail au cours de l'année 1966.

En choisissant un sujet aussi vaste, il est facile de comprendre pourquoi on a tenté à la fois de définir le phénomène de l'automation et de décrire ses implications socio-économiques, au risque de demeurer dans des généralités.

Les exposés, pour la plupart, abordent des points très discutés tels que les effets de l'automation sur l'éducation, la formation professionnelle, l'emploi, le syndicalisme, le climat des relations patronales-ouvrières, etc. En plus de décrire les effets, les conférenciers indiquent les différentes avenues que des recherches subséquentes pourraient emprunter.

Ces exposés théoriques sont suivis de quelques études de cas décrivant avec assez de précision les étapes de l'introduction d'appareils automatiques dans le domaine des communications et de la distribution du gaz et de l'électricité.

Les observations et les réflexions des conférenciers sur les implications de l'automation rejoignent celles des chercheurs nord-américains. Ceci nous amène à conclure que les effets de l'automation demeurent passablement similaires quoique leur intensité varie lorsqu'on passe d'un pays industrialisé à un autre.

C'est un volume qui se lit facilement puisque les exposés sont regroupés sous le thème qui leur est commun. Chaque thème abordé constitue une partie avec des liens établis par des conférenciers et une conclusion rédigée par $M$. Davenport, vice-président de l'Association.

\section{Laurent BELANGER}

Labor Relations and Collective Bargaining, Text and Case, by Max G. Wortman Jr. and George C. Witteried, Boston, Allyn and Bacon, 1969, 388 pp.

Ce livre constitue un instrument pédagogique de première main qui s'inscrit dans les perspectives les plus récentes de l'enseignement en relations industrielles. Il présente, en plus d'un cadre de référence qu'on aurait souhaité un peu plus élaboré, une sélection importante de cas vécus illustrant les différents problèmes qui résultent de la négociation et de l'application de la convention collective.

L'étude et la discussion de ces cas par les étudiants et les professeurs devient un complément intéressant aux enseignements théoriques sur la convention collective en ce qu'ils permettent le contact avec un certain nombre de situations vécues par les parties elles-mêmes.

Il importe de souligner que les cas choisis couvrent une grande variété de problèmes. D'une part, des problèmes que l'on pourrait qualifier de «traditionnels $\gg$ tels que la détermination de l'unité de négociation, la procédure des griefs, les heures de travail, les salaires, etc. D'autre part, on fait place, et c'est là l'un des mérites de l'ouvrage, à des situations qui naissent du développement industriel dans une économie moderne; citons notamment les avantages sociaux, les mesures de protection des salariés en cas de changements technologiques, la question des sous-contrats, etc.

Dans la présentation des «cas», on essaie de reconstituer le mieux possible le contexte dans lequel ils se sont produits. De plus, pour faciliter la discussion, les auteurs proposent une série de questions pertinentes à chaque problème.

S'il s'agit d'un ouvrage à caractère plutôt pratique que théorique, il serait erroné d'affirmer qu'il a été conçu sans référence à des réflexions théoriques. 